\title{
ESTUDO SOBRE OS EFEITOS DA SUBSTITUIÇÃO PARCIAL DO AÇÚCAR POR SUCRALOSE E GOMA XANTANA NAS PROPRIEDADES REOLÓGICAS DAS MASSAS DE BOLOS TIPO ESPONJA
}

\author{
A. V. de O. BARBOSA ${ }^{1}$ e C. E. M. da SILVA ${ }^{1}$ \\ ${ }^{1}$ Universidade Federal do Ceará, Departamento de Tecnologia de Alimentos \\ E-mail para contato: albavaleriaob@yahoo.com.br
}

\begin{abstract}
RESUMO - A qualidade dos bolos relaciona-se com a estrutura aerada, conseguida pela incorporação de ar na massa durante a mistura contribuindo para sua expansão no decorrer do assamento. $\mathrm{O}$ açúcar atua como agente de volume elevando a viscosidade da massa, aumentando a incorporação e retenção do ar, contribuindo para o aumento do volume final, portanto sua redução pode afetar negativamente o fluxo reológico das massas. Objetivou-se avaliar o efeito da substituição crescente do açúcar por sucralose e goma xantana nas propriedades reológicas das massas de bolo tipo esponja, comparando os resultados a uma amostra padrão. O aumento no valor do índice de consistência $(7,08$ $\left.9,87 \mathrm{~Pa} \mathrm{~s}^{\mathrm{n}}\right)$, refletiu na densidade especifica $\left(0,86-0,89 \mathrm{~g} / \mathrm{cm}^{-3}\right)$, indicando boa incorporação de ar, porém os volumes específicos foram menores $\left(1,51-1,05 \mathrm{~cm}^{3} / \mathrm{g}^{-1}\right)$, em relação a amostra controle $\left(1,81 \mathrm{~cm}^{3} / \mathrm{g}\right)$. Uma diminuição mais acentuada da viscosidade durante a mistura, foi relatada como a incapacidade de reter as células de ar durante o assamento, provocando a redução do volume.
\end{abstract}

\section{INTRODUÇÃO}

Entre os produtos de panificação, o bolo vem adquirindo crescente importância no que se refere ao consumo e comercialização, apesar de não constituir alimento básico como o pão, o bolo é aceito e consumido por pessoas de qualquer idade (Borges et al., 2006).

Embora seja um alimento muito apreciado por consumidores em todo o mundo, por apresentar em sua composição altos níveis de açúcar, o mesmo é considerado um alimento muito calórico, estudos revelaram que a ingestão de alimentos ricos em calorias esta ligada ao aumento do número de ocorrências de doenças crônicas como a obesidade e o diabetes (Lee e Lin, 2005). Há uma preocupação crescente por parte da população, principalmente entre obesos e diabéticos, em consumir alimentos de baixo teor energético (Cavalcante, 2012).

Estudos sobre a redução do açúcar datam desde a década de 80, onde se tem demostrado que a redução substancial do açúcar pode ser feita em formulações tradicionais usando uma combinação de um adoçante de alta intensidade e um espessante de baixa caloria (Bennion e Bamford, 1997), pois nenhum agente espessante possui todas as propriedades únicas conferidas ao açúcar, por isso que a 
combinação de agentes de volume deve ser usado como alternativa para a melhoria das características estruturais dos bolos (Baeva et al., 2000).

Os adoçantes artificiais desempenham o papel de fornecimento de doçura. Já os agentes espessantes, são utilizados como agentes de volume que contribuem para o aumento da viscosidade da massa e consequentemente a melhoria da estabilidade e volume dos bolos (Indrani et al., 2012).

No entanto, a redução de níveis de açúcar em formulações de bolo afeta suas propriedades estruturais e sensoriais; o açúcar influencia não apenas no fornecimento de doçura e energia, mas também no controle da formação estrutural dos bolos interferindo diretamente nas propriedades reológicas da massa (Frye e Setser, 1991).

As massas de bolo são uma emulsão complexa óleo em água com uma fase aquosa contínua contendo ingredientes secos dissolvidos ou suspensos. O açúcar atua como agente de volume, mantendo estável a emulsão pois aumenta a viscosidade da massa possibilitando maior retenção das células de ar incorporadas durante a mistura dos ingredientes contribuindo para a estabilidade e um maior volume desejado nos bolos (Schimer et al., 2012). Quando essa viscosidade é muito baixa, a massa não consegue reter as células de ar incorporadas, resultando em bolos de baixo volume (Ronda et al., 2011).

Portanto, compreender o efeito da substituição do açúcar por combinações de adoçantes artificiais e agentes espessantes sobre as características reológicas das massas de bolo é essencial para o desenvolvimento de formulações com reduzido teor de açúcar sem acarretar prejuízos a sua estrutura, já que essas exercem efeitos durante o processamento e sobre as características finais do bolo (Ronda et al., 2011).

Objetivou-se com este trabalho avaliar o efeito da substituição crescente do açúcar por sucralose e goma xantana sobre as propriedades reológicas das massas de bolo tipo esponja, comparando os resultados a uma amostra controle.

\section{MATERIAL E MÉTODOS}

\subsection{Material}

Para o preparo do bolo controle, usado como base comparativa dos resultados obtidos dos bolos com reduzido teor de açúcar, fez-se uso de ingredientes convencionais adquiridos no comércio local (Fortaleza, CE, Brasil): Farinha de trigo Tipo 1 sem fermento, açúcar cristal, margarina sem sal, ovos tamanho médio de cor branca, leite em pó integral, fermento químico em pó e água mineral.

No preparo dos bolos com reduzido teor de açúcar, além dos ingredientes convencionais utilizou-se também o adoçante (sucralose), cedido gentilmente pela TATE \& LYLE (São Paulo-SP, Brasil) e o agente de volume (goma xantana) fornecido pela Gastronomy lab (Brasília-DF, Brasil). 


\subsection{Métodos}

Preparo dos bolos: Para o preparo dos bolos com teor calórico reduzido, utilizou-se uma formulação controle otimizada por Cavalcante (2012), apresentada na Tabela 1. Foram elaboradas 7 (sete) formulações, mantendo fixas as proporções dos ingredientes, farinha de trigo, leite em pó, fermento químico e margarina, variando apenas a quantidade de açúcar no qual foi substituído em reduções crescentes por uma solução conjunta de sucralose a $1 \%$, e goma xantana a 1,5\%. A codificação das formulações baseou-se no nível de substituição do açúcar pela solução de sucralose e goma xantana de acordo com o exposto na Tabela 2, sendo F0, a formulação controle contendo apenas o açúcar na formulação.

Tabela 1- Formulação otimizada

\begin{tabular}{cc}
\hline Ingredientes & Quantidade $(\mathrm{g})$ \\
\hline Farinha & 100 \\
\hline Açúcar & 155,88 \\
\hline Margarina & 28,78 \\
\hline Leite em pó & 6,79 \\
\hline Ovos & 46,96 \\
\hline Fermento químico & 1,00 \\
\hline Água & 59,59 \\
\hline
\end{tabular}

Tabela 2- Formulações de bolos preparadas com substituição do açúcar pela solução conjunta de adoçante e goma xantana

\begin{tabular}{cccc}
\hline $\begin{array}{c}\text { Codificação } \\
\text { das } \\
\text { formulações }\end{array}$ & $\begin{array}{c}\text { Nível de substituição } \\
\text { do açúcar pela solução } \\
\text { adoçante e goma }(\%)\end{array}$ & $\begin{array}{c}\text { Proporção de } \\
\text { açúcar } \\
(\mathrm{g})\end{array}$ & $\begin{array}{c}\text { Proporção de solução } \\
\text { de adoçante e goma } \\
(\mathrm{g})\end{array}$ \\
\hline Controle (F0) & 0,00 & 155,88 & 0,00 \\
\hline F10 & 10,00 & 140,28 & 31,17 \\
\hline F19 & 19,00 & 126,27 & 59,23 \\
\hline F27 & 27,10 & 113,64 & 84,49 \\
\hline F34 & 34,39 & 102,28 & 107,21 \\
\hline F40 & 40,95 & 92,05 & 127,66 \\
\hline F46 & 46,86 & 82,83 & 146,09 \\
\hline F52 & 52,17 & 74,56 & 162,64 \\
\hline
\end{tabular}

Definida as formulações, procedeu-se o preparo dos bolos, utilizando o método rápido de preparo que consiste em adicionar e bater os ingredientes em uma só etapa sem acarretar prejuízos a estrutura do bolo, depois de misturados, os ingredientes foram batidos em batedeira comum (marca, Mallory) com 3 velocidades por 1 (um) minuto na velocidade 1, para permitir a incorporação de ar necessária para o desenvolvimento dos bolos. 


\section{9 a 22 de outubro de 2014 \\ Florianópolis/SC}

Depois de batidos os ingredientes, a massa obtida foi vertida em forma tipo $\mathrm{W}$ e levada para assar em forno elétrico previamente aquecido por 30 minutos a $180^{\circ} \mathrm{C}$, a massa foi assada também a $180^{\circ} \mathrm{C}$ por aproximadamente 40 minutos e resfriadas a temperatura ambiente, e posteriormente armazenados em recipientes plásticos sob refrigeração. Antes de serem levadas para o assamento, amostras das massas foram coletadas, inclusive da controle para análise reológica e densidade especifica. Depois de assados, efetuou-se a medição do volume especifico da fatia dos bolos, segundo metodologia descrita abaixo:

Medidas das propriedades reológicas das massas dos bolos: Para tanto, seguiu-se a metodologia aplicada por Psimouli e Oreopoulou (2012). As análises foram feitas em um reômetro (modelo AR 550, TA instruments), onde aproximadamente $18 \mathrm{~g}$ de massa foram submetidas a uma taxa de cisalhamento de 0,2 a $1 \mathrm{~s}^{-1}$, com controle de temperatura $\left(25^{\circ} \mathrm{C}\right)$ por placa peltier. A viscosidade aparente foi medida em função da taxa de cisalhamento e os dados foram ajustados ao Modelo de Ostwald-de-Waele (Lei da potência), $\mu=K \times \gamma^{n}$, onde $\mu$ é a viscosidade aparente (Pa s), K é o coeficiente de consistência $\left(\mathrm{Pa}^{\mathrm{n}}\right)$ e $\gamma$ é a taxa de cisalhamento $\left(\mathrm{s}^{-1}\right)$ e $\mathrm{n}$ é o índice de fluxo. Para o ajuste dos dados utilizou-se o software TA Advantage Data Analysis, versão 5.0.38, do próprio equipamento. Analise feita em triplicata.

Característica física referente à Densidade especifica das massas dos bolos: Foi medida gravimetricamente, pela razão entre um recipiente padrão contendo $100 \mathrm{~mL}$ da massa e o peso do mesmo recipiente padrão contendo $100 \mathrm{~mL}$ de água segundo metodologia adotada por Lin e Lee (2005). Analise em triplicata.

Característica física alusivo ao volume especifico do bolo: Depois de assado os bolos, efetuouse o quarteamento para melhor representatividade da amostra, efetuando em seguida a medição do volume especifico da fatia dos bolos segundo metodologia descrita pela AACC (2000), por deslocamento da semente de painço. Análise feita em triplicata.

Tratamento estatístico: Os resultados obtidos com as análises da massa antes e depois do assamento foram submetidas à análise estatística, para tanto, usou-se o Delineamento Inteiramente Casualizado (DIC), onde foram feitas análises de variância (ANOVA) e cálculo do desvio das médias com posterior comparação das diferenças entre as médias pelo teste de Tukey a um nível de significância de $95 \%$ ( p<0,05), utilizando o software Statistica versão 8.

\section{0-RESULTADO E DISCUSSÃO}

A Figura 1 apresenta o reograma com as curvas da viscosidade aparente em função da taxa de cisalhamento das amostras de massa do bolo com reduzido teor calórico, incluindo a amostra controle (F0), para fins comparativos. Observa-se que, todas as amostras incluindo a controle apresentaram um fluxo pseudoplástico, ou seja, a viscosidade aparente diminuiu com o aumento da taxa de cisalhamento. Os valores obtidos com o índice de fluxo que variaram de 0,49 a 0,27, (Tabela 3) confirmam esse resultado, visto que para $\mathrm{n}<1$ o fluxo é pseudoplástico e, $\mathrm{n}>1$, é dilatante (Toneli et al., 2004). 

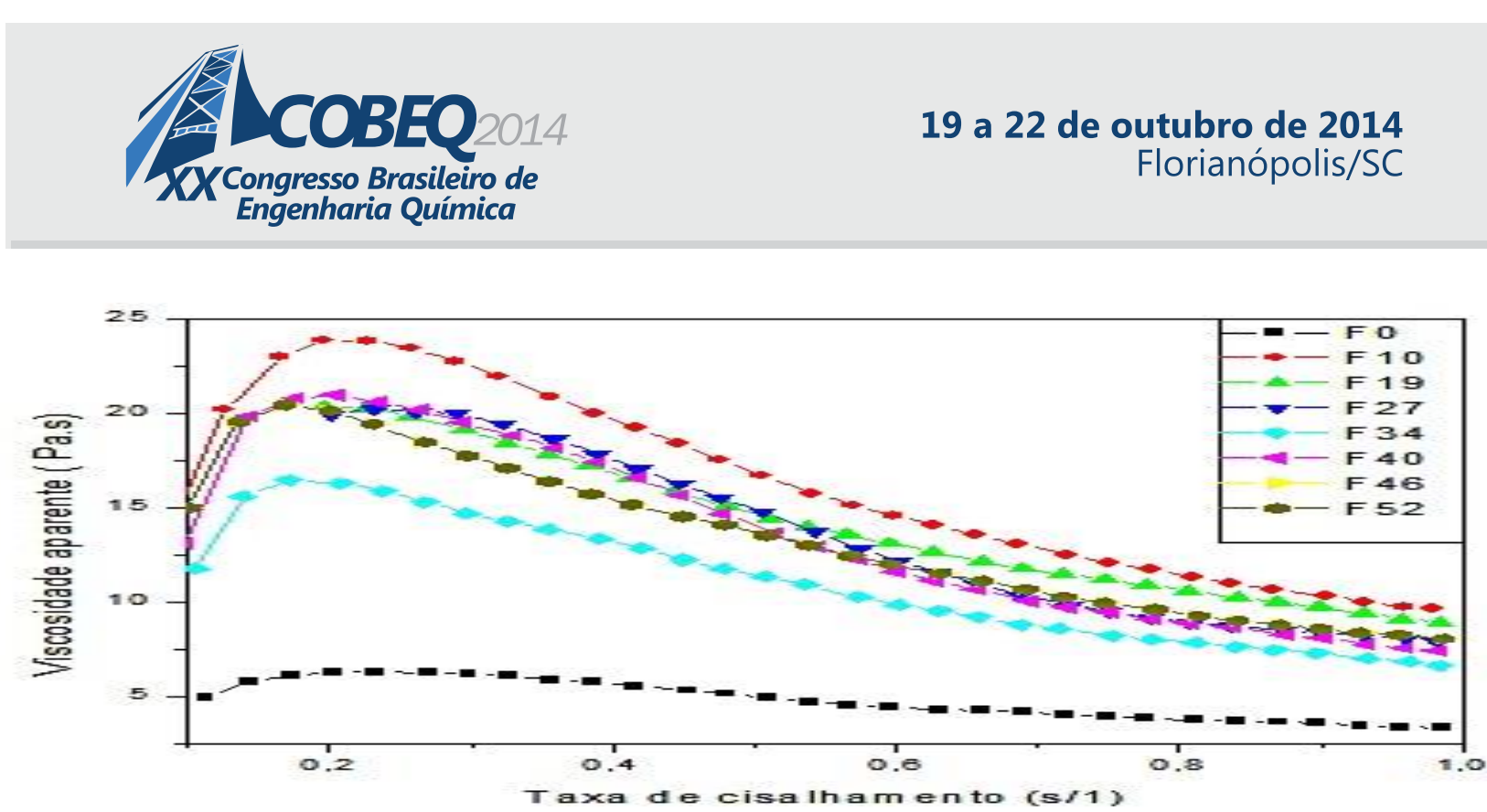

Figura 1 -Reograma apresentando as curvas de viscosidade aparente em função da taxa de cisalhamento das massas de bolo com substituição parcial do açúcar por uma solução de sucralose e goma xantana.

Os valores das medidas reológicas de coeficiente de consistência $\left(\mathrm{P}\right.$ as $\left.{ }^{\mathrm{n}}\right)$ segundo modelo da Lei da potência são apresentados na Tabela 3. As amostras de bolo com substituição parcial do açúcar pela solução de sucralose e goma xantana não diferiram significativamente entre si, exceto a formulação F34 em relação à F52. Todas as formulações apresentaram valores entre 7,08-9,87 Pa s , superiores a controle $\left(3,15 \mathrm{P}^{\mathrm{n}}{ }^{\mathrm{n}}\right)$.

Segundo Shiroma (2012), o coeficiente de consistência K, indica o grau de resistência da amostra ao escoamento ou fluxo, ou seja, quanto maior o valor de $\mathrm{K}$ maior a sua resistência ao escoamento e, portanto, maior a sua viscosidade aparente.

A viscosidade é uma importante propriedade física, uma vez que está intimamente relacionada com a qualidade do produto final. Ela também é um dos fatores que controlam o seu volume no final do processo de assamento (Lee et al., 2004). Os trabalhos de Lee et al., 2004, Martínez-Cervera et al., 2012 e Psimouli e Oreopoulou, 2012 mostraram que quando a viscosidade é elevada, essa proporciona o aumento da incorporação e retenção das células de ar a massa durante a mistura dos ingredientes, contribuindo para um maior volume e estabilidade dos bolos, indicada por uma menor densidade específica da massa.

A densidade específica fornece uma indicação do total de ar incorporado e retido na massa durante a mistura. Valores baixos indicam uma boa incorporação de ar, obtendo-se um volume final elevado depois do assamento (Martínez-Cervera et al., 2012).

Os valores obtidos com a densidade específica para as amostras da massa dos bolos com substituição parcial do açúcar pela solução de sucralose e goma xantana expostos na Tabela 3, variaram entre $0,86-0,89 \mathrm{~g} / \mathrm{cm}^{-3}$, não diferindo significativamente entre si e a amostra controle $\left(0,86 \mathrm{~g} / \mathrm{cm}^{-3}\right)$. Tais valores são um indicativo de que ouve uma boa incorporação de ar a massa. 
No entanto, os resultados obtidos para o volume específico dessas amostras de bolo com substituição parcial do açúcar pela solução de sucralose e goma xantana, que variaram entre 1,90$1,06 \mathrm{~cm}^{3} / \mathrm{g}^{-1}$ (Tabela 3) foram inferiores a controle $\left(1,81 \mathrm{~cm}^{3} / \mathrm{g}^{-1}\right)$ excetuando as formulações F10 e F19 que não diferiram significativamente entre si e a controle. Segundo Psimouli e Oreopoulou (2011), o volume específico pode ser utilizado como um indicador do desenvolvimento de volume dos bolos.

O comportamento viscoso apresentado pelas amostras de massa dos bolos com substituição parcial do açúcar pela solução de sucralose e goma xantana permitiu uma boa incorporação de ar a massa, contudo os baixos valores de volume específico apresentados, sugerem deficiência na expansão da massa durante o assamento.

Resultado semelhante foi encontrado por Lin e Lee (2005), em seus estudos sobre os efeitos da substituição parcial do açúcar por sucralose e dextrina em bolos. Onde a formulação com $80 \%$ de substituição do açúcar por sucralose e dextrina, apresentou a mesma capacidade de incorporação de ar a massa $\left(0,49 \mathrm{~g} / \mathrm{cm}^{3}\right)$ que a formulação controle $\left(0,45 \mathrm{~g} / \mathrm{cm}^{3}\right)$. No entanto o seu volume específico $\left(1,36 \mathrm{~cm}^{3} / \mathrm{g}^{-1}\right)$, também foi inferior ao obtido na controle $\left(1,81 \mathrm{~cm}^{3} / \mathrm{g}^{-1}\right)$. Eles relataram ainda que os bolos obtidos a partir dessa formulação apresentaram baixa estabilidade e volume reduzido.

Tabela 3-Valores das medidas reológicas, coeficiente de consistência e índice de fluxo e características físicas referente a densidade especifica da massa e volume especifico do bolo

\begin{tabular}{ccccc}
\hline Formulações & $\begin{array}{c}\text { Coeficiente de } \\
\text { consistência } \\
\left(\mathrm{P} \mathrm{as}^{\mathrm{n}}\right)\end{array}$ & $\begin{array}{c}\text { Índice de } \\
\text { fluxo } \\
(\mathrm{n})\end{array}$ & $\begin{array}{c}\text { Densidade } \\
\text { Especifica } \\
\left(\mathrm{g} / \mathrm{cm}^{-3}\right)\end{array}$ & $\begin{array}{c}\text { Volume especifico } \\
\left(\mathrm{cm}^{3} / \mathrm{g}^{-1}\right)\end{array}$ \\
\hline F0 & $3,15^{\mathrm{c}} \pm 0,32$ & $0,49^{\mathrm{a}} \pm 0,00$ & $0,86^{\mathrm{a}} \pm 0,00$ & $1,81^{\mathrm{a}} \pm 0,01$ \\
\hline F10 & $9,69^{\mathrm{ab}} \pm 0,29$ & $0,35^{\mathrm{b}} \pm 0,05$ & $0,86^{\mathrm{a}} \pm 0,03$ & $1,90^{\mathrm{a}} \pm 0,09$ \\
\hline F19 & $8,53^{\mathrm{ab}} \pm 1,02$ & $0,36^{\mathrm{b}} \pm 0,04$ & $0,88^{\mathrm{a}} \pm 0,01$ & $1,80^{\mathrm{a}} \pm 0,03$ \\
\hline F27 & $8,62^{\mathrm{ab}} \pm 0,97$ & $0,27^{\mathrm{bc}} \pm 0,04$ & $0,86^{\mathrm{a}} \pm 0,02$ & $1,51^{\mathrm{b}} \pm 0,05$ \\
\hline F34 & $7,08^{\mathrm{b}} \pm 0,30$ & $0,29^{\mathrm{b}} \pm 0,06$ & $0,87^{\mathrm{a}} \pm 0,01$ & $1,19^{\mathrm{c}} \pm 0,03$ \\
\hline F40 & $7,40^{\mathrm{ab}} \pm 0,28$ & $0,25^{\mathrm{c}} \pm 0,05$ & $0,87^{\mathrm{a}} \pm 0,00$ & $1,17^{\mathrm{c}} \pm 0,01$ \\
\hline F46 & $7,80^{\mathrm{ab}} \pm 0,57$ & $0,32^{\mathrm{b}} \pm 0,02$ & $0,87^{\mathrm{a}} \pm 0,00$ & $1,16^{\mathrm{c}} \pm 0,02$ \\
\hline F52 & $9,87^{\mathrm{a}} \pm 0,97$ & $0,33^{\mathrm{b}} \pm 0,04$ & $0,89^{\mathrm{a}} \pm 0,01$ & $1,05^{\mathrm{c}} \pm 0,03$ \\
\hline
\end{tabular}

Os resultados são apresentados como valores médios \pm desvio padrão. Valores seguidos por letras sobrescritas diferentes na mesma coluna são significativamente diferentes $(p<0,05)$ onde $a>b>c$ 


\section{9 a 22 de outubro de 2014 \\ Florianópolis/SC}

Isso pode ser justificado pelos baixos valores do índice de fluxo (n) das formulações de bolo com substituição parcial do açúcar exibidos na Tabela 3, que variaram entre 0,25-0,36 em relação a controle $(0,49)$. Sugerindo que essas amostras, foram mais susceptíveis ás deformações provocadas pelo aumento da taxa de cisalhamento.

Essa maior susceptibilidade à deformação ocasionou a redução demasiada da viscosidade aparente das massas dos bolos, que segundo Sarabjit e Alava (2003), possibilita a incorporação adequada de ar a massa. Contudo, isso não implica que a capacidade de retenção das células de ar não tenha sido afetada.

Sugerindo que, durante o assamento as formulações com substituição parcial do açúcar por sucralose e goma xantana apresentaram deficiência capacidade de retenção das células de ar. $\mathrm{O}$ que permitiu o escape dessas células de ar para a superfície do bolo restringindo assim expansão da massa, resultando em bolos de menor volume.

As formulações F10 e F19 obtiveram resultados reológicos e físicos semelhantes a controle. Isso significa que nesses níveis de substituição do açúcar não a prejuízos as propriedades reológicas da massa e do volume final dos bolos.

\section{0-CONCLUSÃO}

As características reológicas da massa são fatores de controle das características de qualidade do bolo, no que concerne o desenvolvimento do volume desejado. Com base nisso, conclui-se que a substituição crescente do açúcar pela solução de sucralose e goma xantana afetou negativamente as propriedades reológicas da massa e consequentemente a formação estrutural dos bolos. Mesmo apresentando em todas as formulações com substituição parcial do açúcar um comportamento viscoso superior a formulação controle, que possibilitou igual incorporação de ar a massa. Os baixos valores de índice de fluxo sugeriram deficiência na retenção das células de ar incorporadas que são responsáveis pela expansão da massa durante o assamento, resultando em bolos de menor volume.

\section{0- REFERENCIAS}

AACC International. Approved methods of the AACC. $10^{\text {th }}$ ed. AACC The Association: St Paul, Minnesotta, 2000.

BAEVA, M. R.; PANCHEV, I. N.; TERZIEVA, V. V. Comparative study of texture of normal and energy reduced sponge cakes. Narhung, v. 44, p.22-26, 2000.

BENNION, E.B.; BAMFORD, G. S. T. The Technology of Cake Making. London: Blackie Academic e Professional, 1997.

BORGES, J. T. S.; PIROZI, M. R.; VIDIGAL, J. G.; Paula, D. C. de.; SILVA, N. A. S. de. Utilização de farinha mista de aveia e trigo na elaboração de bolos. R. Brasileira Tecno.l Agroin., v. 24, p. 145-162, 2006. 
CAVALCANTE, R. S. Avaliação das características estruturais de bolos com redução calórica. Dissertação (Mestrado em Ciência e Tecnologia de Alimentos). Universidade Federal do Ceará, Fortaleza, 2012.

FRYE, A. M., SETSER, C.S. Optimizing texture of reduced-calorie yellow layer cakes. Cereal Chem., v. 29, p. 338-343. 1991.

INDRANI, D. Studies on interaction between stevioside, liquid sorbitol, hydrocolloids and emulsifiers for replacement of sugar in cakes. Food Hyd. v.29, n. 2, 2012.

LEE, S.; INGlETT, G. E.; CARRIERE, C. Effect of Nutrim Oat Bran and Flaxseed on Rheological Properties of Cakes. Cereal Chem., v. 81, p. 22-33. 2004.

LIN, S-D.; LEE, C-C. Qualities of chiffon cake prepared with indigestible dextrin and sucralose as replacement for sucrose. Cereal Chem., v. 82, p. 405-413, 2005.

MARTÍNEZ-CERVERA, S.; SANZ, T.; FISZMAN, SM. Rheological, textural and sensorial properties of low-sucrose muffins reformulated with sucralose/polydextrose. LWT - Food Sci and Technol., V. 45, n. 2, p. 213-220, 2012.

PSIMOULI, V.; OREOPOULOU, V. The effect of alternative sweeteners on batter rheology and cake properties. J. sci Food Agric., v. 92, p. 99-105, 2012.

RONDA, F.; OLIETE, B. GOMEZ, CABALlERO, P. A., PANDO, V. Rheological study of layer cake batters made with soybean protein isolate and different starch sources. J. Food Eng, v. 102, p.272-277, 2011.

SARABJIT, S.; ALAVA, J. M. Functionality of emulsifiers in sponge cake Production. J. Sci Food Agric., v. 83, p.1419-1429, 2003.

SCHIRMER, M. Physicochemical interactions of polydextrose for sucrose replacement in pound cake. Food Rearch Inter. V. 49, 2012.

SHIROMA, P. H. Estudo do comportamento reológico de suspensões aquosas de bentonita e CMC: Influência da concentração de $\mathrm{NaCl}$. Dissertação (Mestrado em Engenharia Química). Universidade de São Paulo, São Paulo, 2012.

TONELI, J.T. de C. L.; MURR, F. E. X.; PARK, K. J. Estudo da reologia de polissacarídeos utilizados na indústria de alimentos. R. Brasileira de P. Agroin. v.7, p. 181-204, 2005. 